\title{
Ablation of the PTHrP gene or the PTH/PTHrP receptor gene leads to distinct abnormalities in bone development
}

\author{
Beate Lanske, ${ }^{1}$ Michael Amling, ${ }^{2}$ Lynn Neff, Jennifer Guiducci, ${ }^{1}$ \\ Roland Baron, ${ }^{3}$ and Henry M. Kronenberg ${ }^{1}$ \\ ${ }^{1}$ Endocrine Unit, Massachusetts General Hospital and Harvard Medical School, Boston, Massachusetts 02114, USA \\ ${ }^{2}$ Department of Bone Pathology, University of Hamburg, D-20146 Hamburg, Germany \\ ${ }^{3}$ Department of Cell Biology, Yale University School of Medicine, New Haven, Connecticut 06510, USA
}

Address correspondence to: Henry M. Kronenberg, Endocrine Unit, Wellman 501, Massachusetts General Hospital, 50 Blossom Street, Boston, Massachusetts 02114, USA. Phone: (617) 726-3966; Fax: (617) 726-7543; E-mail: kronenberg.henry@mgh.harvard.edu.

Beate Lanske's present address is: Molecular Endocrinology, Max-Planck-Institut für Biochemie, 82152 Munich, Germany.

Received for publication February 23, 1999, and accepted in revised form July 13, 1999.

\begin{abstract}
Parathyroid hormone (PTH) and parathyroid hormone-related peptide (PTHrP) bind to and activate the same PTH/PTHrP receptor. Deletion of either the PTHrP gene or the PTH/PTHrP receptor gene leads to acceleration of differentiation of growth plate chondrocytes. To explore further the functional relationships of PTHrP and the PTH/PTHrP receptor, bones of knockout mice were analyzed early in development, and the phenotypes of double-knockout mice were characterized.

One early phenotype is shared by both knockouts. Normally, the first chondrocytes to become hypertrophic are located in the centers of long bones; this polarity is greatly diminished in both these knockouts. The PTH/PTHrP receptor-deficient $\left(P T H / P T H r P-R^{-/}\right)$mice exhibited 2 unique phenotypes not shared by the $\mathrm{PTHrP} \mathrm{P}^{-/-}$mice. During intramembranous bone formation in the shafts of long bones, only the $P T H / P T H r P-R^{-/-}$bones exhibit a striking increase in osteoblast number and matrix accumulation. Furthermore, the $P T H / P T H r P-R^{-/-}$mice showed a dramatic decrease in trabecular bone formation in the primary spongiosa and a delay in vascular invasion of the early cartilage model. In the doublehomozygous knockout mice, the delay in vascular invasion did not occur. Thus, PTHrP must slow vascular invasion by a mechanism independent of the $\mathrm{PTH} / \mathrm{PTH} \mathrm{P}$ receptor.
\end{abstract}

J. Clin. Invest. 104:399-407 (1999).

\section{Introduction}

The formation of the skeleton depends on the differentiation, function, and interaction of various cell types. Two distinct developmental patterns occur. Most bones are formed by endochondral ossification, in which a cartilage mold is replaced by bone. First, mesenchymal cells condense and differentiate into chondrocytes. The chondrocytes in the center of the shaft become hypertrophic and synthesize a distinct extracellular matrix that rapidly becomes mineralized. Osteoclasts then resorb this matrix, allowing blood vessel invasion, and osteoblasts bind to the cartilaginous matrix remnants and deposit bone matrix around them. Later, the remnants are removed and replaced by bone matrix. At the ends of the bone, the epiphyseal growth plates are formed when chondrocytes nearest the ends of bones proliferate and then undergo hypertrophy. A second process by which bone is formed is intramembranous ossification, in which matrix is deposited directly in the mesenchyme by osteoblasts, without replacement of a cartilage matrix. In the long bones, bone collar osteoblasts first participate in intramembranous ossification in the mesenchyme adjacent to the most hypertrophic chondrocytes.

Many signaling systems control bone formation. The PTH/PTHrP receptor is at the center of one such system. This receptor integrates signals from both the calcium- regulating hormone parathyroid hormone $(\mathrm{PTH})$ and the paracrine factor parathyroid hormone-related protein (PTHrP). PTH is known to influence bone formation in various ways (1). In cultured cells, PTH decreases the production of type I collagen (2) and can enhance the synthesis of noncollagenous proteins, including osteocalcin $(3,4)$ and interstitial collagenase (collagenase 3 ; ref. 5). PTH can decrease both the proliferation of osteoblasts (6) and the formation of bone nodules from osteoprogenitor cells in vitro (7). On the other hand, in vivo PTH can increase bone formation, particularly when administered intermittently, and in trabecular bone (8). While the effects of PTHrP on osteoblasts are poorly characterized, the effects of PTHrP on growth plate chondrocytes have been demonstrated by the phenotype of the PTHrP $P^{-/-}$mouse (9). This phenotype shows that PTHrP slows the differentiation of chondrocytes in the growth plate (10).

The PTH/PTHrP receptor is found in cells of the kidney tubule and in chondrocytes and osteoblasts of bone (11), and thus might mediate many actions of both PTH and PTHrP. However, other putative receptors for both ligands have been found (12-17), and one has been cloned (18). That the PTH/PTHrP receptor mediates the calcium-regulating action of PTH has been demonstrated by the inability of PTH to stimulate ${ }^{45}$ Ca release from calvariae 
of PTH/PTHrP receptor-deficient $\left(P T H / P T H r P-R^{-/-}\right)$mice (19). That the same receptor mediates the action of PTHrP to slow differentiation of chondrocytes has been shown by the similar accelerated differentiation of chondrocytes in the growth plates of $P T H r P^{-/-}$and $P T H / P T H r P-R^{-/-}$mice at E18.5 (20). Furthermore, mice missing either PTHrP or the PTH/PTHrP receptor are resistant to the action of Indian hedgehog (Ihh), which slows chondrocyte differentiation by stimulating production of PTHrP $(10,20)$.

Nevertheless, as might be expected for a system involving multiple ligands and receptors, $\mathrm{PTHrP}^{-/-}$and PTH/PTHrP- $\mathrm{R}^{-/-}$mice do not have identical phenotypes $(19,21)$. Some differences may derive from the ability of PTH to compensate for the loss of PTHrP in the PTHrP $P^{-/-}$mouse but not in the PTH/PTHrP- $R^{-/-}$mouse. For example, the collagenase $3 \mathrm{mRNA}$ in osteoblasts of $\mathrm{PTHrP}^{-/-}$mice is normal, but is very low in PTH/PTHrP$R^{-/-}$mice (19). Other differences, like the low placental calcium transport found in $P T H r P^{-/-}$mice but not in $P T H / P T H r P-R^{-/-}$mice, are probably explained by unique receptors for $\mathrm{PTHrP}(21)$.

Because of the dramatic effects of both PTHrP and $\mathrm{PTH} / \mathrm{PTHrP}$ receptor gene ablation on the growth plates of endochondral bones $(9,20)$, we undertook a more detailed analysis of the earlier development of chondrocytes and osteoblasts in PTH/PTHrP- $\mathrm{R}^{-/-}$and $\mathrm{PTHrP} \mathrm{P}^{-/}$ mice. We found that (a) PTHrP and the PTH/PTHrP receptor are needed for the normal polarity of early chondrocyte maturation in the cartilage mold; (b) the $\mathrm{PTH} / \mathrm{PTHrP}$ receptor regulates the production of osteoblasts in the primary spongiosa and in the bone collar differently (receptor activation decreases the formation of diaphyseal cortical bone, and increases the formation of trabecular bone in the primary spongiosa), because receptor ablation leads to an increase in osteoblasts in the bone collar and a decrease in osteoblasts in the primary spongiosa; and (c) the $\mathrm{PTH} / \mathrm{PTHrP}$ receptor accelerates, and PTHrP delays, the invasion of blood vessels into hypertrophic cartilage; this observation emphasizes the functional complexity of the PTH/PTHrP network of ligands and receptors.

\section{Methods}

Mice. Mice carrying either a disrupted PTH/PTHrP receptor gene $\left(P T H / P T H r P-R^{-/-}\right)$or a disrupted PTHrP gene $\left(P T H r P^{-/}\right)$were derived by homologous recombination in embryonic stem cells of 129SVJ genetic background, as described previously $(9,20)$. Because the survival rate of $\mathrm{PTH} / \mathrm{PTH} \mathrm{H} P \mathrm{R}^{-/-}$mice until birth was increased when they were backcrossed 1 generation into the outbred Black Swiss strain, we backcrossed the PTHrP knockout mice for 1 generation into the same strain, in order to provide a comparable genetic background for the performed studies. To obtain double-knockout mice that are disrupted for both the PTHrP gene and the PTH/PTHrP receptor gene (PTHrP $P^{-/} ;$PTH/PTHrP- $\mathrm{R}^{-/-}$), we mated double-heterozygous males and females with each other and performed cesarean sections at 18.5. All mice were given a standard chow diet and water. Genomic DNA was isolated from tail clips to genotype mice by standard methods described previously $(9,20,21)$. These studies were approved by the Institutional Animal Care and Use Committee of Massachusetts General Hospital.

Fixation, tissue preparation, and histology. Fresh tissues were obtained by cesarean section from fetuses at different days of gestation after timed matings. Regular histology, in situ hybridization, paraffin embedding, and von Kossa staining were performed as described previously (19, 22-24). All slides were interpreted by at least 2 observers unaware of the genotypes of the particular mice being examined.

For methylmethacrylate embedding, bone samples were dissected out after autopsy and fixed in 3.7\% buffered formalin for 18 hours at $4^{\circ} \mathrm{C}$. After dehydration, the undecalcified bones were embedded in methylmethacrylate, and $5-\mu \mathrm{m}$ sections were prepared on a rotation microtome as described previously $(25,26)$. Sections were stained with toluidine blue, von Kossa stain, or Goldner trichrome.

\section{Results}

Early bone development in PTH/PTHrP- $\mathrm{R}^{-/-}$and $\mathrm{PTHr} \mathrm{P}^{-/-}$ mice. In previous reports, we characterized the phenotypic abnormalities in the tibial growth plates of $\mathrm{PTHrP}^{-/-}$and $\mathrm{PTH} / \mathrm{PTHrP}-\mathrm{R}^{-/}$mice late in gestation (E18.5). In both cases, these include the shortness of proliferative columns of chondrocytes, the diminished synchrony of chondrocyte differentiation across the growth plate $(9,20,27,28)$, and glycogen accumulation in epiphyseal chondrocytes (19). To understand better how these abnormalities develop, we examined bones at earlier gestational time points. Figure 1 compares phalanges of $P T H / P T H r P-R^{-/-}$and wild-type mice at E15.5. In the normal phalanx, chondrocytes in the center of the bone have hypertrophied, and synthesize type X collagen mRNA. In contrast, only the earliest hint of differentiation has occurred in the center of the phalanx of the $P T H / P T H r P-R^{-/-}$mouse, and no type $\mathrm{X}$ collagen mRNA can yet be detected (Figure 1). This delay in chondrocyte differentiation is accompanied by a partial loss in polarity of the axis of chondrocyte differentiation across the length of the bone; this loss in polarity is demonstrated best at a somewhat later stage of differentiation, at E16.5 (Figure 2). As in Figure 1, the hypertrophic chondrocytes in the PTH/PTHrP- $R^{-/-}$phalanx are small, and now produce small amounts of type $\mathrm{X}$ collagen mRNA. Strikingly, whereas the hypertrophic chondrocytes are concentrated in the center of the wild-type phalanx, the moderately hypertrophic chondrocytes in the $P T H / P T H r P-R^{-/-}$phalanx extend from the center to encompass most of the bone. In the same bone from a $\mathrm{PTHrP}^{-/-}$mouse, the same partial loss of polarity of differentiation is observed. In contrast to the PTH/PTHrP$R^{-/-}$bone, however, there is no obvious delay in initial chondrocyte hypertrophy or reduction of type X collagen mRNA in the $\mathrm{PTHrP} \mathrm{P}^{-/-}$bone.

Chondrocyte hypertrophy is normally followed by blood vessel invasion and deposition of bone by osteoblasts. Figure 3 a illustrates this process in a normal (wild-type) phalanx at E18.5. In contrast, the hypertrophic chondrocytes in the center of the PTH/PTHrP$R^{-/-}$phalanx have been incompletely replaced by blood vessels and bone (Figure 3b). Figure 3, d and e compare tibiae at a roughly analogous developmental time 
(E16.5). Again, the center of the normal tibia demonstrates vascular invasion and bone deposition, while the PTH/PTHrP- $R^{-/-}$tibia shows no vascular invasion or bone deposition. Figure 3, $\mathrm{c}$ and $\mathrm{f}$ also show that vascular invasion and bone deposition are not delayed in the $\mathrm{PTHrP}^{-/-}$bones; in fact, these processes appear more advanced than in normal bones. Analogous studies in the xiphoid across the genotypes revealed the same patterns found in the long bones (data not shown).

These studies of early bone development demonstrate that $P T H / P T H r P-R^{-/-}$bones exhibit a partial loss in polarity of the normal gradient of cellular differentiation from the center to the end of the bone. They also exhibit a delay in chondrocyte differentiation, followed by a delay in blood vessel invasion and bone deposition. The $\mathrm{PTHrP}^{-/-}$mice share the loss in polarity of differentiation, but exhibit no delay in chondrocyte differentiation, blood vessel invasion, or bone deposition.

Abnormal bone formation in PTH/PTHrP- $R^{-/}$and $P T H r P^{-/-}$mice. The abnormalities in chondrocyte development in the PTH/PTHrP- $R^{-/-}$mice were accompanied by abnormalities in osteoblast development. In the intramembranous bone adjacent to hypertrophic chondrocytes (illustrated by the metaphyseal region of the tibia [Figure 4, a and b] and the diaphyseal region of a phalanx [Figure 4, $\mathrm{d}$ and e], both at E18.5), the $\mathrm{PTH} / \mathrm{PTHr}-\mathrm{R}^{-/-}$bones unexpectedly revealed multiple layers of osteoblasts and woven bone matrix instead of the normal 1-2 layers of osteoblasts. Also, the matrix of the PTH/PTHrP- $R^{-/-}$bone demonstrated a delay in mineral deposition (black areas, von Kossa staining), whereas the $\mathrm{PTHrP}^{-/-}$mice show the opposite - an accelerated mineral deposition at the same site (Figure $4, \mathrm{c}$ and $\mathrm{f}$. The increase in metaphyseal intramembranous bone in the $\mathrm{PTH} / \mathrm{PTH} \mathrm{P} \mathrm{P}^{-\mathrm{R}^{-/}}$mice persists as a strikingly thickened diaphyseal cortex in long bones such as the tibia and fibula, shown in cross-section in Figure 5, and in longitudinal section in Figure $6 \mathrm{~b}$ (both at E18.5). In contrast, the intramembranous bone of the $\mathrm{PTHrP}^{-/-}$bones (Figure 4, c and $\mathrm{f}$ ) showed no consistent abnormality in osteoblast number, matrix deposition, or mineralization. Analogous patterns were shown in the xiphoid across the genotypes (data not shown).

In contrast to the intramembranous bone, the bone in the primary spongiosa, which is laid down on the matrix produced by hypertrophic chondrocytes after vascular invasion, is dramatically diminished in the bones of PTH/PTHrP- $\mathrm{R}^{-/-}$mice (Figure 6). Correspondingly, all of the long bones of these mice exhibit little trabecular bone throughout the metaphyseal and diaphyseal regions (data not shown). In contrast, the primary spongiosa and trabecular bone of the $P T H r P^{-/-}$bones differ little from wild-type bones (data not shown).

Bones of double-homozygous PTHrP ${ }^{-/} ;$PTH/PTHrP-R $R^{-/}$ embryos. The contrasting patterns of chondrocyte differentiation, vascular invasion, and bone deposition in the PTH/PTHrP- $R^{-/-}$and $P T H r P^{-/-}$mice probably derive from the complexity of the signaling network in which the PTH/PTHrP receptor and PTHrP participate. It may be that PTH or other ligands compensate for the lack of $\mathrm{PTHrP}$ in $\mathrm{PTHrP} \mathrm{P}^{-/}$mice, but could not compensate for the lack of the PTH/PTHrP receptor. Alternatively, PTHrP might act on other receptors to generate phenotypes distinct from those controlled by the PTH/PTHrP receptor. To evaluate these alternatives, we examined the paws and xiphoids of 5 double-homozygous mice at E18.5, and compared them in blinded fashion to the bones of $5 \mathrm{PTHrP}^{-/-}$and $5 \mathrm{PTH} / \mathrm{PTHrP}^{-\mathrm{R}^{-/-}}$mice.
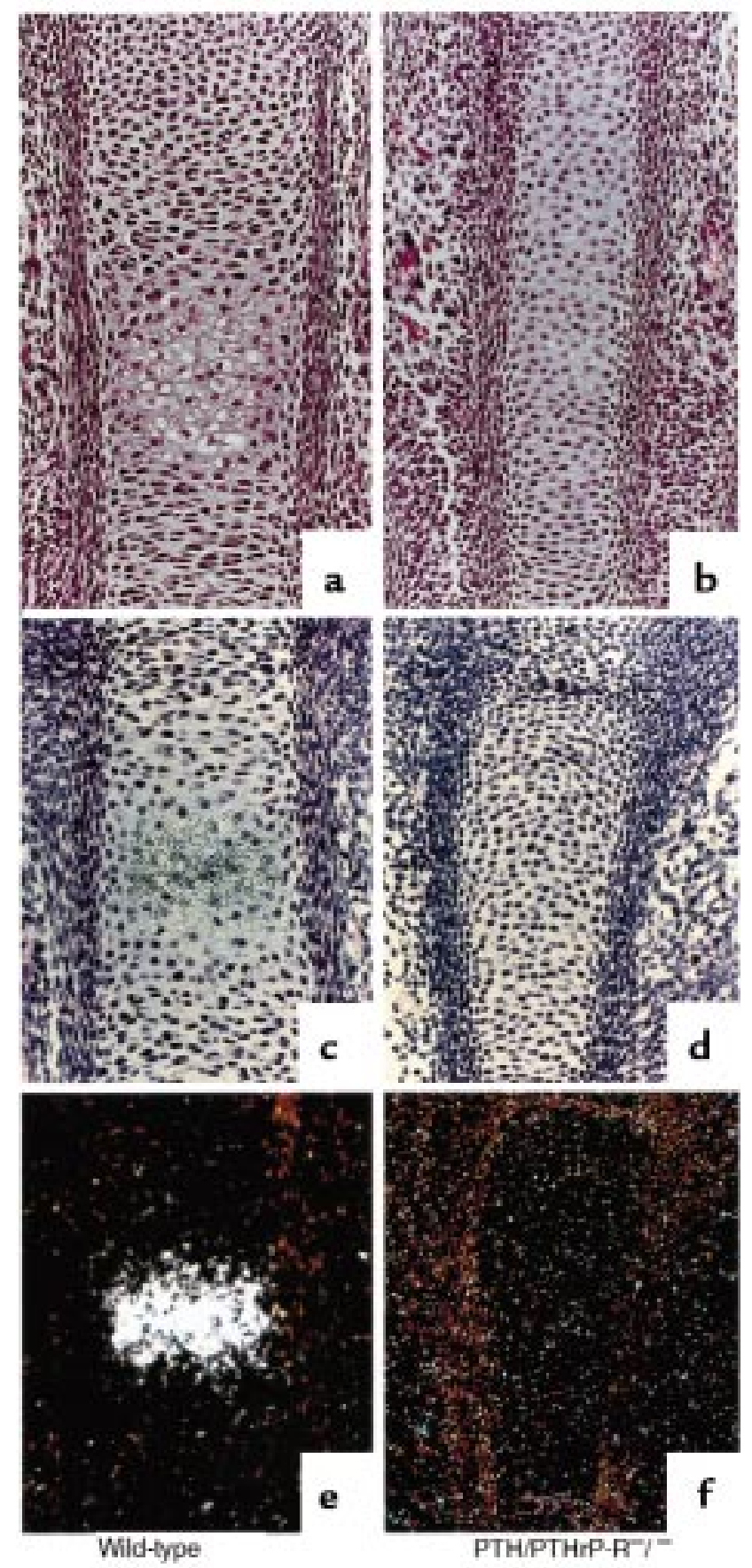

Figure 1

Chondrocyte differentiation at E15.5. Hematoxylin/eosin staining (a and b) and type $X$ collagen mRNA in situ hybridization ( $\mathbf{c}-\mathbf{f}$ ) of wild-type and PTH/PTHrP-R-/- phalanges. PTH/PTHrP receptor-ablated bones show a delay in chondrocyte differentiation and type $X$ collagen expression $(\mathbf{b}, \mathbf{d}$, and $\mathbf{f}$ ) when compared with wild-type bones (a, $\mathbf{c}$, and $\mathbf{e}$ ). Bright-field, $\mathbf{c}$ and $\mathbf{d}$; dark-field, $\mathbf{e}$ and $\mathbf{f}$. 

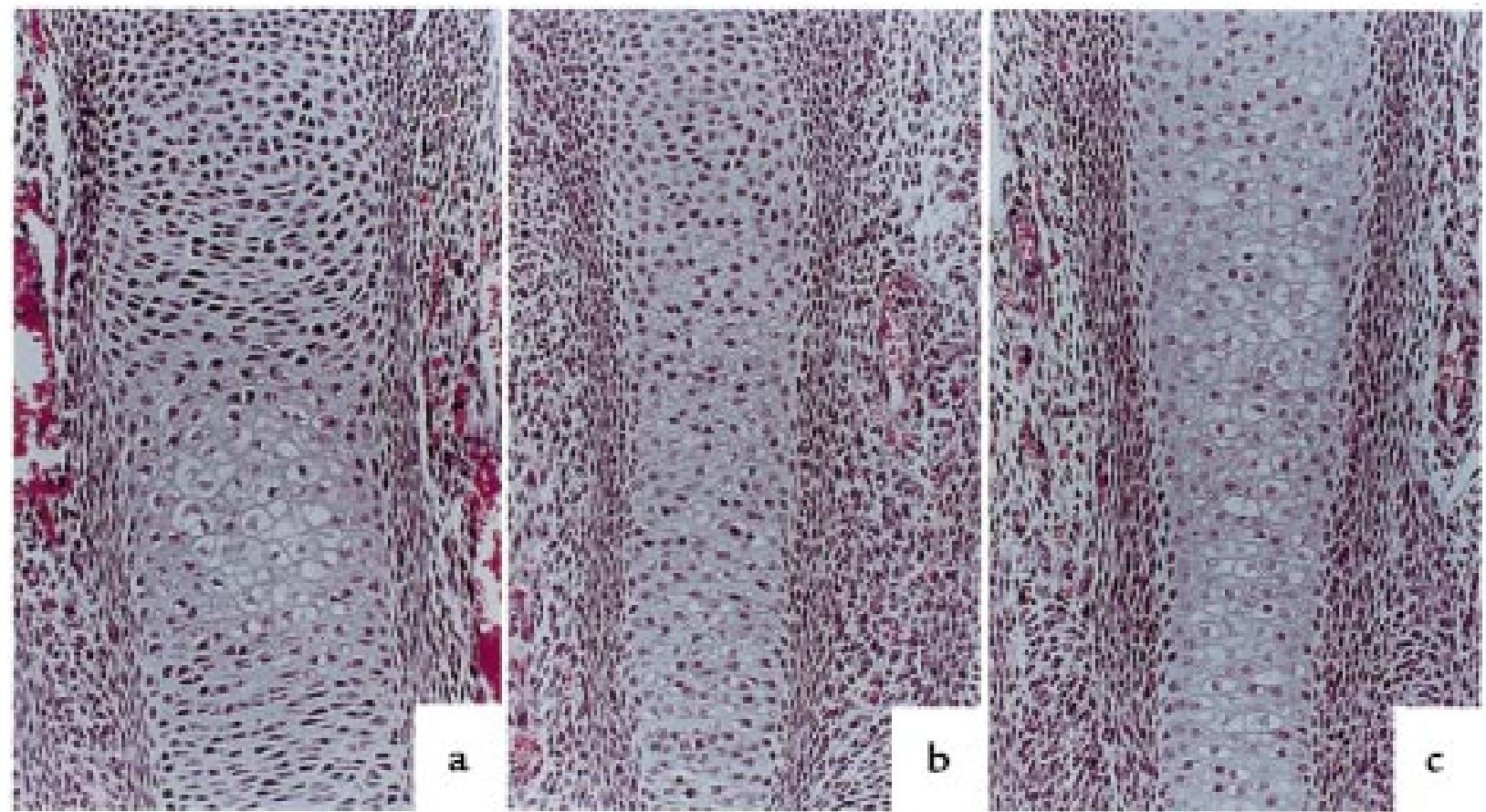

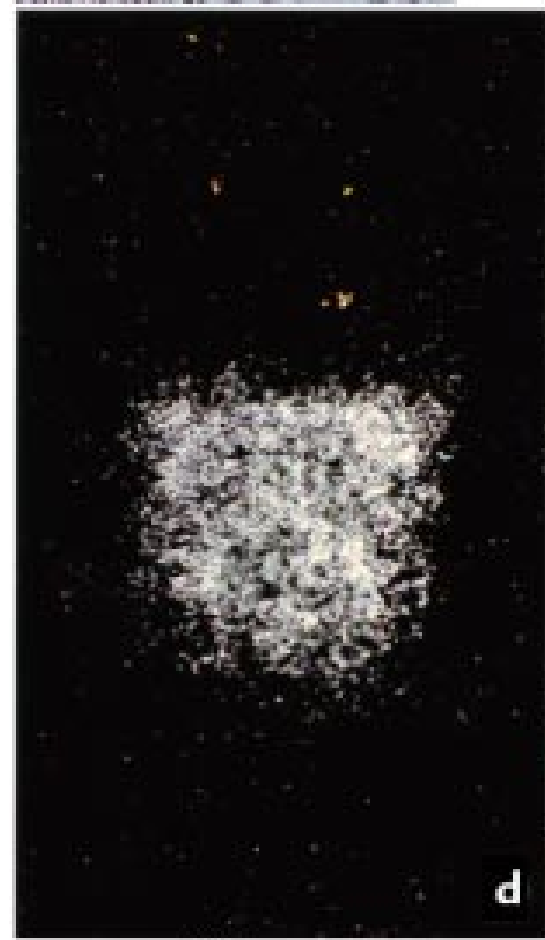

Wild-typo

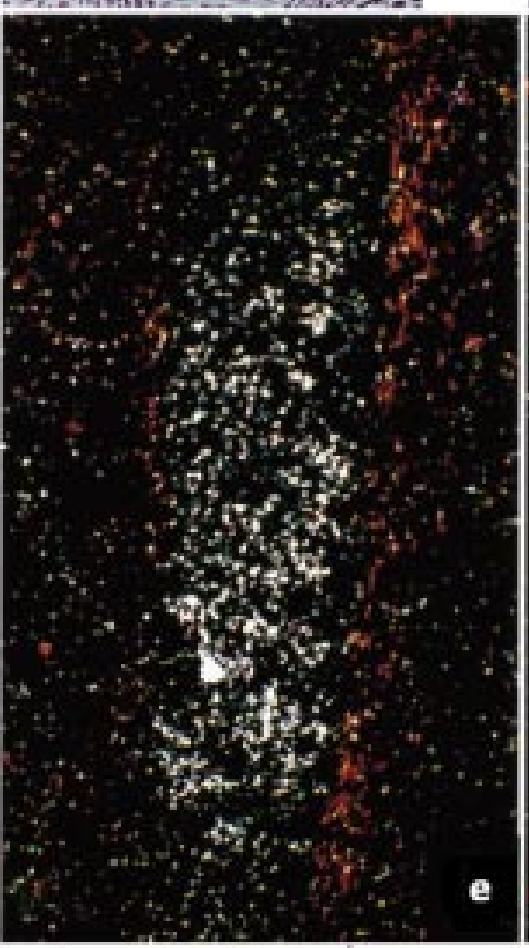

PTHIPTHIP, ? $^{-}$

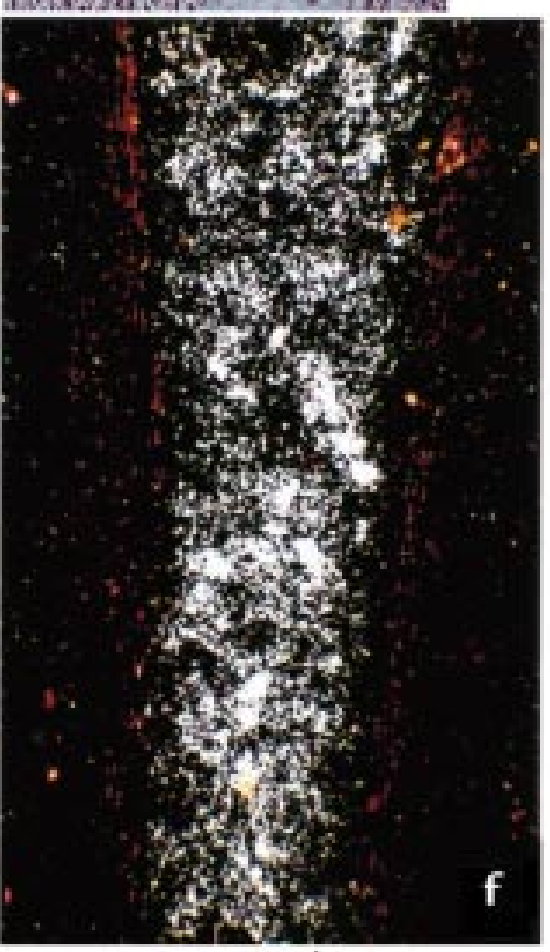

PTHipl-

\section{Figure 2}

Chondrocyte differentiation at E16.5. Hematoxylin/eosin staining (a-c) and type X collagen mRNA in situ hybridization (d-f) of wild-type, PTH/PTHrP-R $R^{-/-}$, and $P T H r P^{-/-}$phalanges at E16.5. In contrast to PTHrP $P^{-/-}$bones, which exhibit a spatial and temporal progression in chondrocyte differentiation ( $\mathbf{c}$ and $\mathbf{f}$ ), $P T H / P T H r P-R^{-/-}$bones show a delay in chondrocyte differentiation, as shown by the decrease in type $\mathrm{X}$ collagen expression ( $\mathbf{b}$ and $\mathbf{e}$ ), when compared with wild-type bones ( $\mathbf{a}$ and $\mathbf{d}$ ). However, the spatial distribution of the abnormally differentiated hypertrophic cells is identical (b, $\mathbf{c}$ and $\mathbf{e}, \mathbf{f})$. 

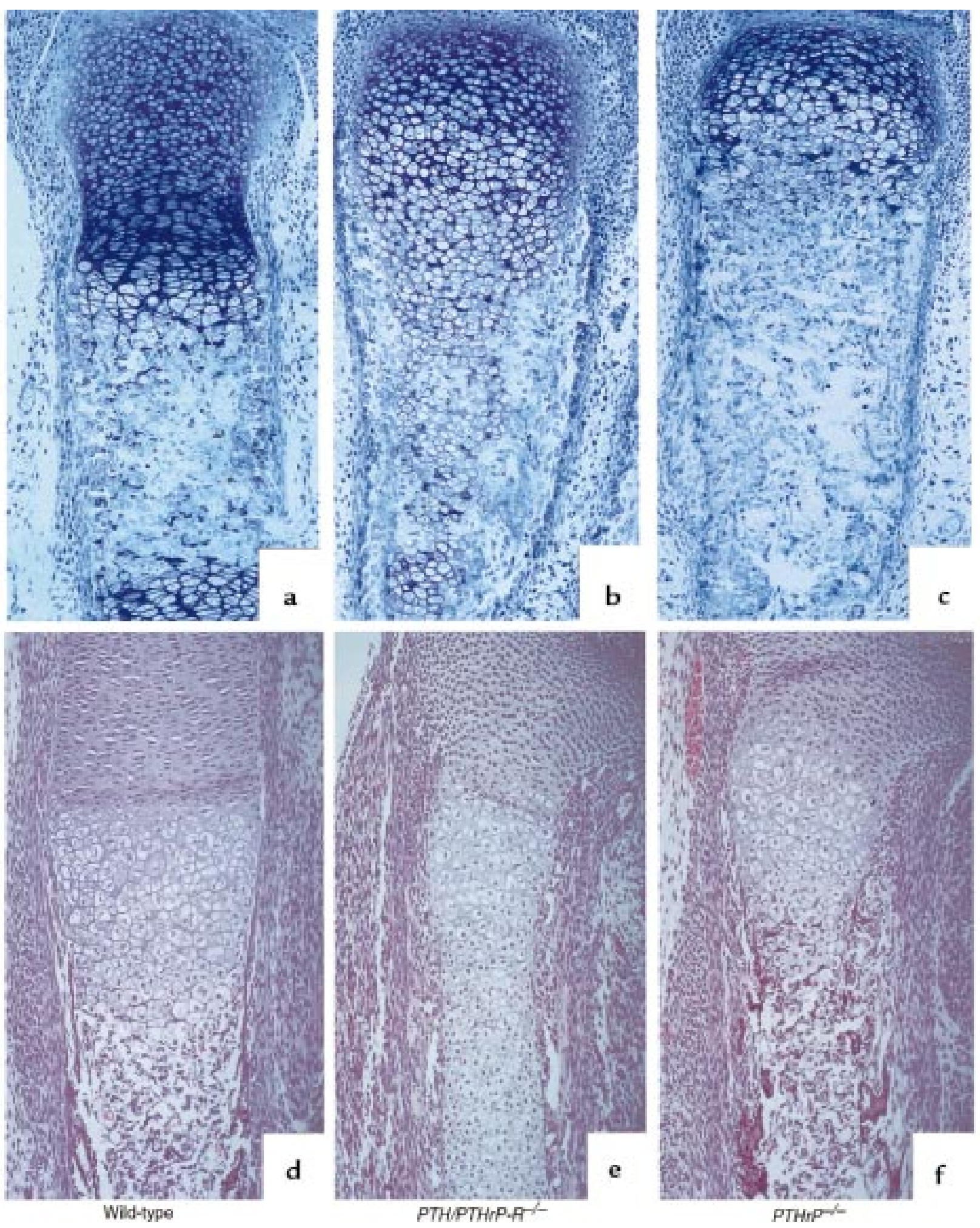

Figure 3

Delay in replacement of cartilage by bone. Methylmethacrylate sections of phalanges at E18.5 (a-c) and paraffin sections of tibiae at E16.5 (d-f) of wild-type, $P T H / P T H r P-R^{-/-}$, and $P T H r P^{-/-}$animals. Blood vessel invasion and deposition of bone by osteoblasts are delayed in $P T H / P T H r P-R^{-/-}$bones (b and $\mathbf{e}$ ) when compared with wild-type bones ( $\mathbf{a}$ and $\mathbf{d}$ ). In contrast, in $P T H r P^{-1-}$ bones, these processes are advanced ( $\mathbf{c}$ and $\mathbf{f}$ ). 
Consistently, the double-homozygous mice exhibited a partial rescue of the delay in blood vessel invasion observed in the PTH/PTHrP- $R^{-/-}$mice (Figure 7). In contrast, the enhanced intramembranous bone formation with multiple layers of cortical osteoblasts seen in the PTH/PTHrP- $R^{-/-}$mice was also seen in the doublehomozygous mice.

\section{Discussion}

The growth plates of E18.5 $\mathrm{PTHrP}^{-/-}$and $\mathrm{PTH} / \mathrm{PTHrP}$ $R^{-/-}$mice exhibit similar accelerated differentiation of chondrocytes, diminished synchrony of differentiation, and resistance to effects of Ihh on chondrocyte differentiation $(9,10,20)$. These results suggest that PTHrP acts on the PTH/PTHrP receptor to regulate the rate and synchrony of differentiation of chondrocytes in a feedback loop in which Ihh participates $(10,20)$. Here we have examined earlier stages of bone development. We have found further abnormalities in chondrocyte and osteoblast development in the PTH/PTHrP- $R^{-/}$mice, and have found that many of these abnormalities are not shared by $\mathrm{PTHrP} \mathrm{P}^{-/-}$mice. These findings emphasize that the PTH/PTHrP receptor responds to at least 2 different ligands, and that the ligands may have more than 1 signaling mechanism (summarized in Figure 8).

Both the PTH/PTHrP-R $\mathrm{R}^{-/}$and $\mathrm{PTHrP}^{-/-}$bones exhibit abnormalities in the polarity of chondrocyte differentiation in long bones. Normally, chondrocytes in the center of a bone (farthest from the ends of the bone) differentiate first into hypertrophic chondrocytes. As these cells are replaced by blood vessels, marrow elements, and osteoblasts, cells progressively closer to the ends of the bone become hypertrophic. In both $P T H / P T H r P-R^{--}$and PTHrP $P^{-1-}$ mice, this polarity is disrupted. The first cells to become hypertrophic are in the center of the bone, but very quickly the transition to hypertrophic differentiation spreads, so that cells near the ends of the bone and near the center are similarly hypertrophic. Polarity is not completely lost, however, and growth plates form in their normal locations near the ends of the bone once vascular invasion takes place. It seems likely that the normal polarity of PTHrP mRNA expression, which is greatest near the ends of bones, particularly early in development (22), is partly responsible for the normal polarity of chondrocyte differentiation that is disrupted in the mutant mice.

In contrast to the chondrocytes in $\mathrm{PTHrP}^{-/-}$mice,
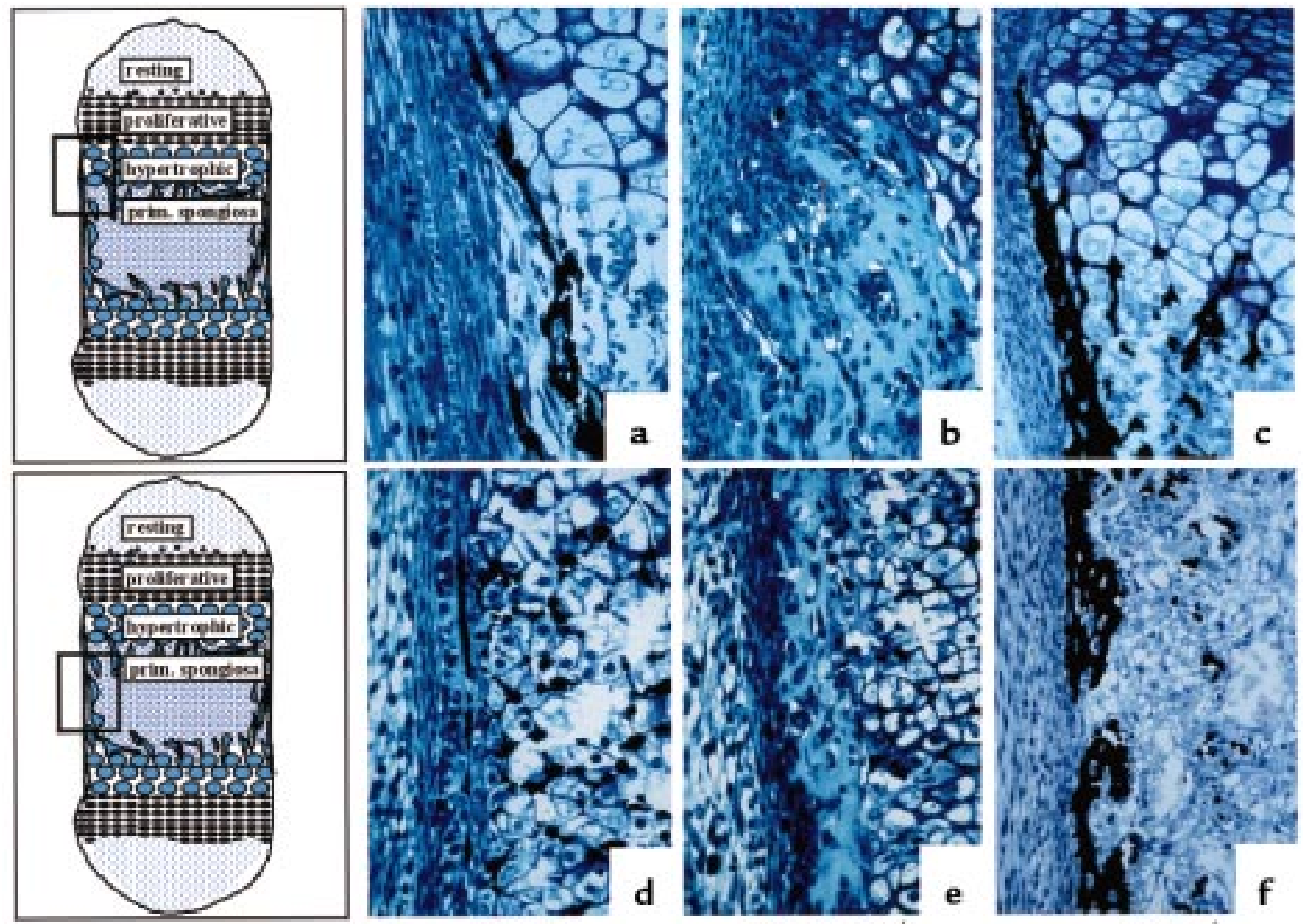

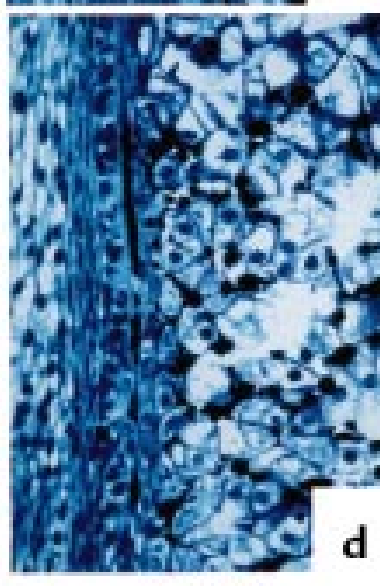

Wid-type

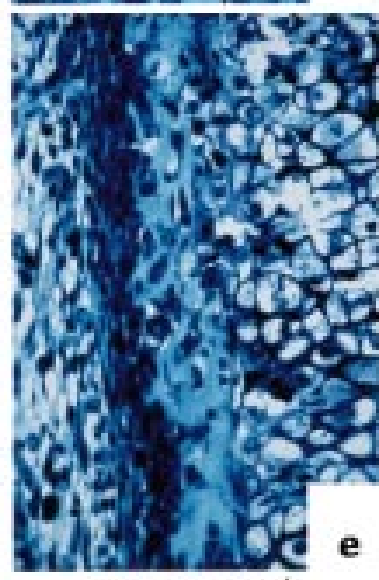

PTHPTHIP.F ${ }^{\prime-}$

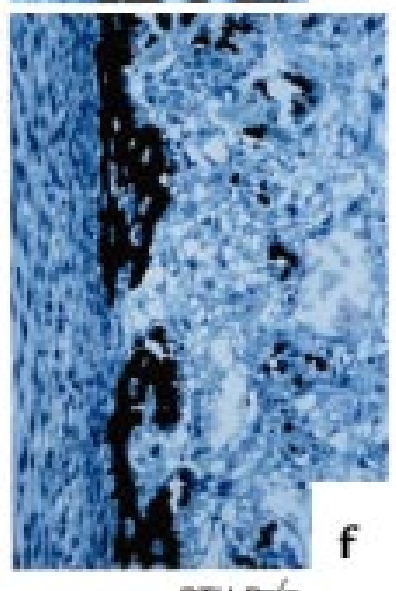

PTHiP\&-

\section{Figure 4}

Increase in osteoblast number and cortical bone in $P T H / P T H r P-R^{-1-}$ animals. Von Kossa staining on methylmethacrylate sections at the level of the metaphyseal region of a tibia (a-c) and at the diaphyseal region of a phalanx (d-f) in wild-type, $P T H r P^{-/-} ; P T H / P T H r P-R^{-/-}$, and $P T H r P^{-/-}$animals at E18.5. PTH/PTHrP receptor mutant bones reveal an abnormal augmentation in osteoblast layers accompanied by an increased bone matrix ( $\mathbf{b}$ and e) that does not mineralize, as demonstrated by the lack of von Kossa staining. In contrast, PTHrP-/- bones (c and f) look indistinguishable or somewhat advanced in terms of mineralization and replacement of cartilage by bone when compared with wild-type bones (a and $\mathbf{d}$ ). 
Figure 5

Increase in cortical bone in PTH/PTHrP- $R^{-} /$ embryos. Transverse section of tibia and ulna of E18.5 wild-type (a) and PTH/PTHrP-R-/ embryos (b). The abnormal increase in cortical bone in the mutant is clearly indicated by the black brackets.
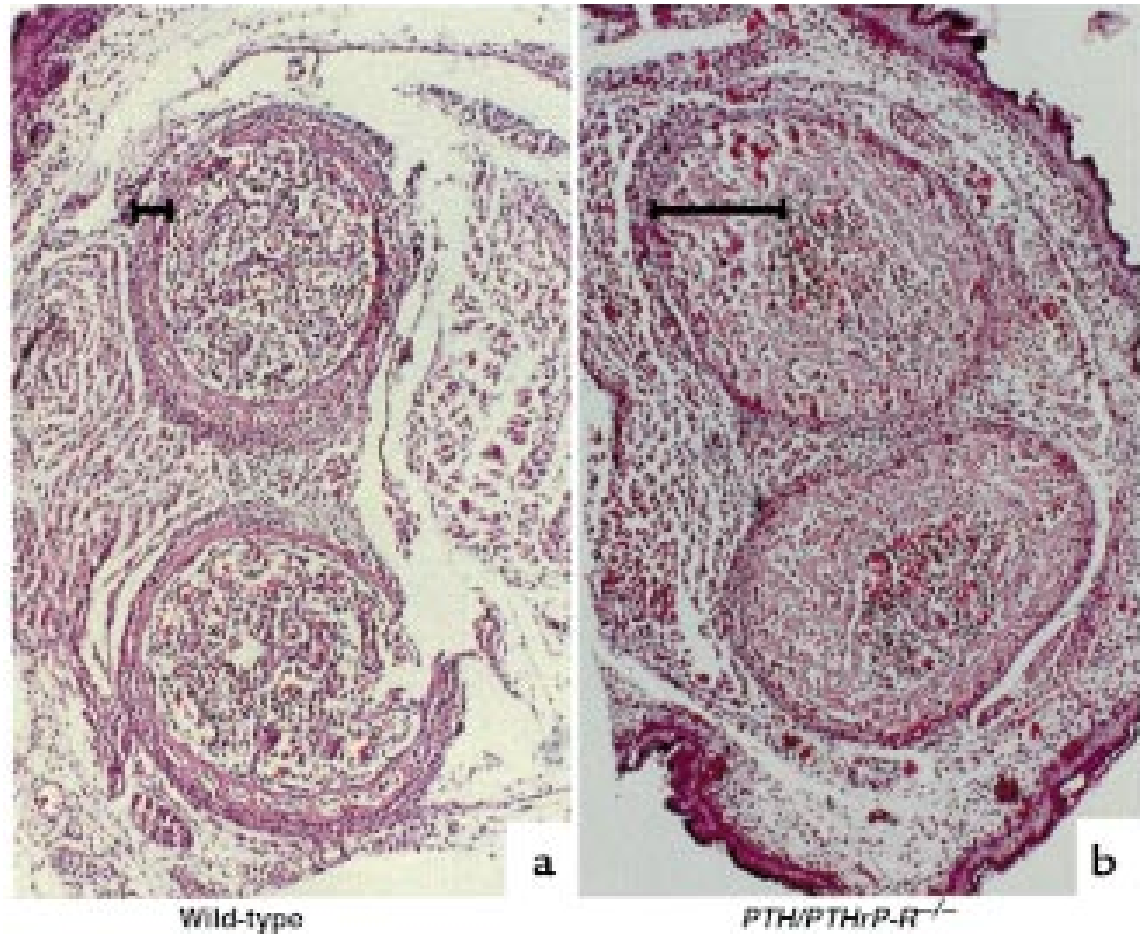

chondrocytes in the centers of developing bone in $\mathrm{PTH} / \mathrm{PTHr} P-\mathrm{R}^{-/-}$mice become hypertrophic later than in wild-type mice. Again in contrast to $\mathrm{PTHr} \mathrm{P}^{-/-}$mice, after hypertrophy, these chondrocytes are replaced by invading blood vessels and osteoblasts more slowly than in wild-type mice. The primary spongiosa, which is the first bone to form on a cartilage matrix, is greatly diminished in PTH/PTHrP- $\mathrm{R}^{-/-}$mice; consequently, this leads to a decrease in secondary spongiosa, with less trabecular bone throughout the skeleton. None of these abnormalities are found in the bones of $\mathrm{PTHrP}^{-/-}$mice. The delay in cell differentiation specific to the $P T H / P T H r P-R^{-/}$ bones could be explained by (a) the inability of PTH to compensate for the lack of PTHrP action on the $\mathrm{PTH} / \mathrm{PTHrP}$ receptor; (b) effects of the increased levels of PTH observed in these animals (C. Kovacs et al., unpublished observations) on a distinct PTH-specific receptor; or (c) effects of PTHrP independent of the PTH/PTHrP receptor. Additional receptors for PTH (14, $16,18)$ and PTHrP $(13,29,30)$ have been described or postulated to explain actions of the ligands. In addition, PTHrP may have direct nuclear actions (31).

To distinguish between these hypotheses, the bones of double-homozygous $\left(\mathrm{PTHr}^{-/-} ; \mathrm{PTH} / \mathrm{PTHr} \mathrm{P}^{-\mathrm{R}^{-/}}\right)$mice

\section{Figure 6}

Decrease in trabecular bone in PTH/PTHrP-R $R^{-/-}$tibia. Von Kossa staining of a wild-type (a) and a PTH/PTHrP-R $R^{-/}$(b) tibia at E18.5. Increase in cortical bone and dramatic diminution in trabecular bone (arrow) are shown in $\mathbf{b}$.
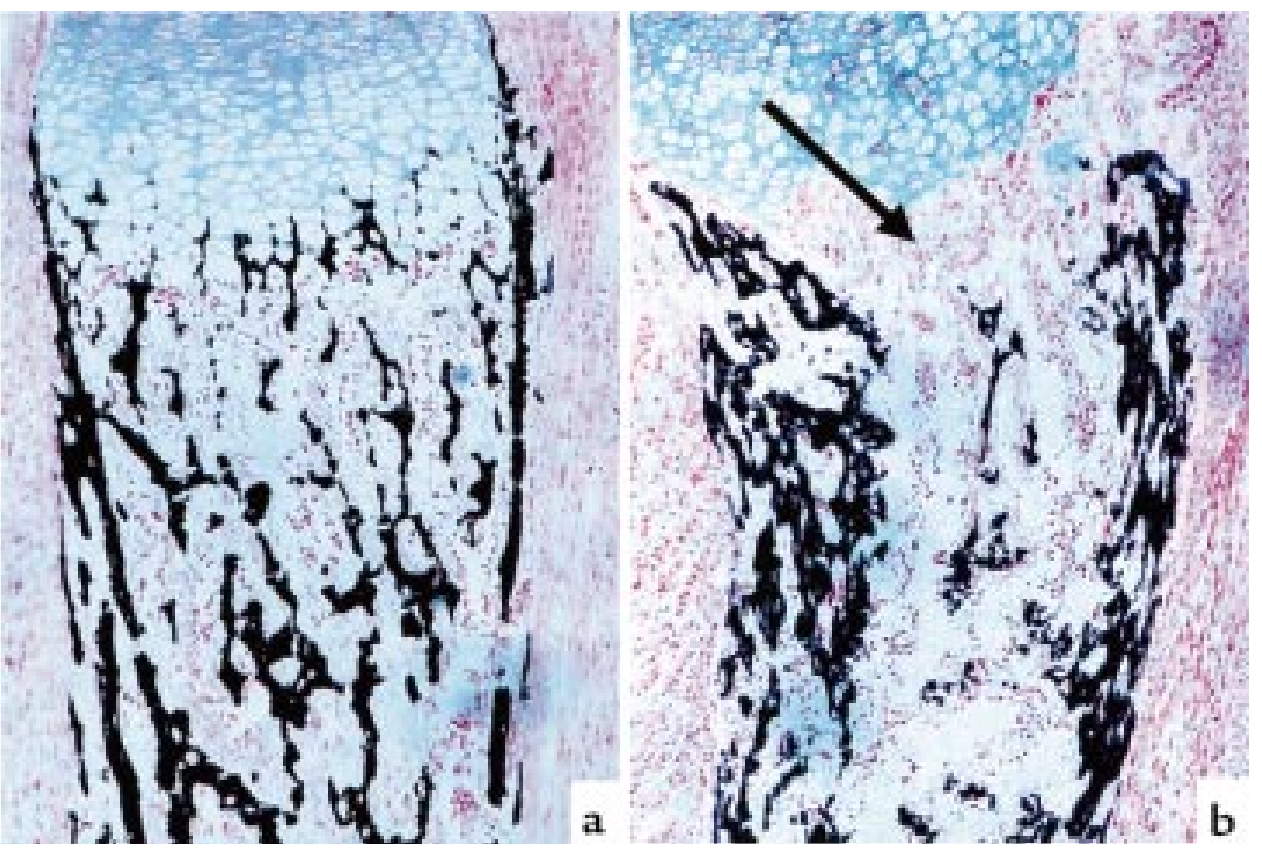


\section{Figure 7}

Partial rescue of vascular invasion in bones of double-homozygous mice. Hematoxylin/eosin staining of a PTH/PTHrP-R $R^{-/-}$(a) and a $P T H r P^{-1-} ; P T H / P T H r P-R^{-/-}$doublehomozygous (b) phalanx at E18.5. The additional ablation of PTHrP from the PTH/PTHrP receptor gene knockout bone leads to a partial rescue of the delay in vascularization. Note the red blood cells amidst the chondrocytes in the double mutants (b).

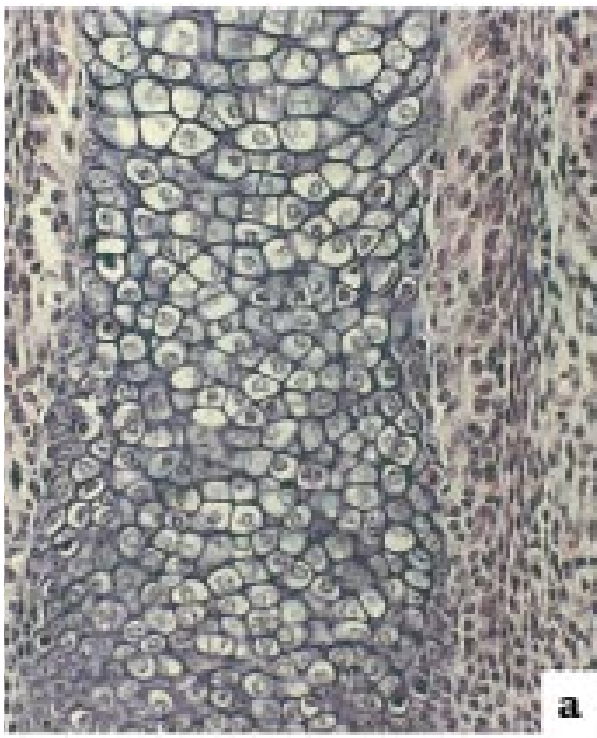

PTHPTHIP.PT'-

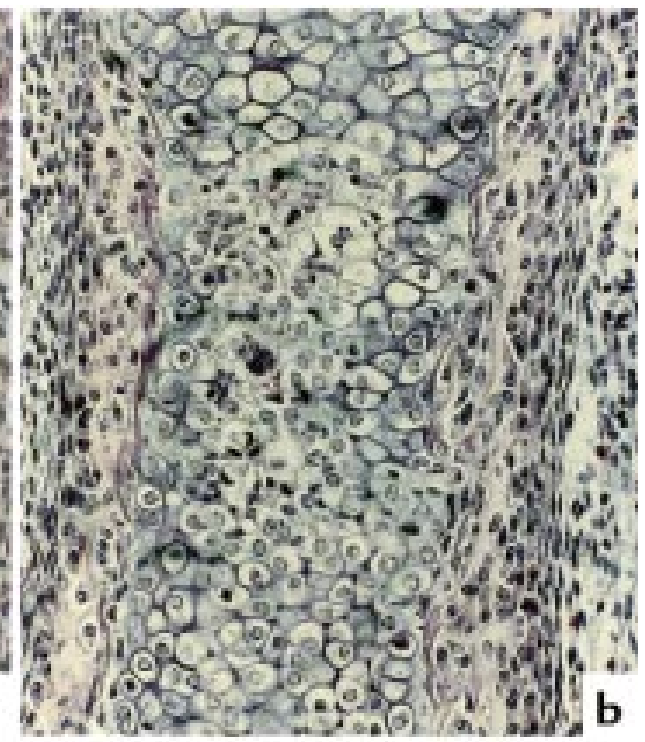

PTHRP-ATHPTHIP.A- were examined at E18.5. These bones demonstrated vascular invasion and endochondral bone formation closer to wild-type than were exhibited by the PTH/PTHrP-R-/bones. Thus, deletion of the PTHrP gene partially rescues the phenotype of $P T H / P T H r P-R^{-/-}$mice. Therefore, some action of PTHrP independent of activation of the $\mathrm{PTH} / \mathrm{PTHrP}$ receptor must explain the phenotype of the double-homozygous mice. PTHrP, acting perhaps through a distinct receptor or through nuclear actions, may slow blood vessel and osteoblast invasion, an effect particularly obvious in $P T H / P T H r P-R^{-/-}$mice.

In contrast to the paucity of bone in the primary spongiosa, the intramembranous bone adjacent to differentiating chondrocytes in PTH/PTHrP- $\mathrm{R}^{-/-}$bones contains abundant matrix and multiple layers of osteoblasts. Because identical findings are exhibited in the
PTHrP-/-PTH/PTHrP-R $\mathrm{R}^{-/-}$bones, the absence of these observations in $P T H r P^{-/-}$bones may well result from the action of PTH to compensate for the action of the missing PTHrP. The increase in osteoblast number in the absence of the PTH/PTHrP receptor may reflect the action of PTH to suppress bone nodule formation in vitro (32). The increased matrix in the PTH/PTHrP- $R^{-/-}$ bones may reflect the action of PTH to decrease type I collagen synthesis in vitro (2). $P T H / P T H r P-R^{-/}$mice, but not $P \mathrm{THrP}^{-/-}$mice, also exhibit decreased expression of osteopontin, osteocalcin, and collagenase 3 mRNA (19).

The differing effects of the $\mathrm{PTH} / \mathrm{PTHr}$ receptor mutation on the development of trabecular and cortical bone underscore the differential regulation of the osteoblasts involved in endochondral and intramembranous bone formation. It is tempting to relate these observations to the

\section{Figure 8}

Pathways of action of PTH and PTHrP. The top bone cartoon represents early long bone development, with the first differentiation of hypertrophic chondrocytes limited to the central region. This polarity of differentiation is minimal in early stages of development in the PTH/PTHrP$R^{-/-}$mice. Vascular invasion of the bone is slowed by actions of PTHrP independent of those of the PTH/PTHrP receptor. The bottom bone cartoon illustrates the cortical bone produced by intramembranous formation, and the trabecular bone. Actions of the PTH/PTHrP receptor decrease intramembranous formation and increase formation of trabecular bone.

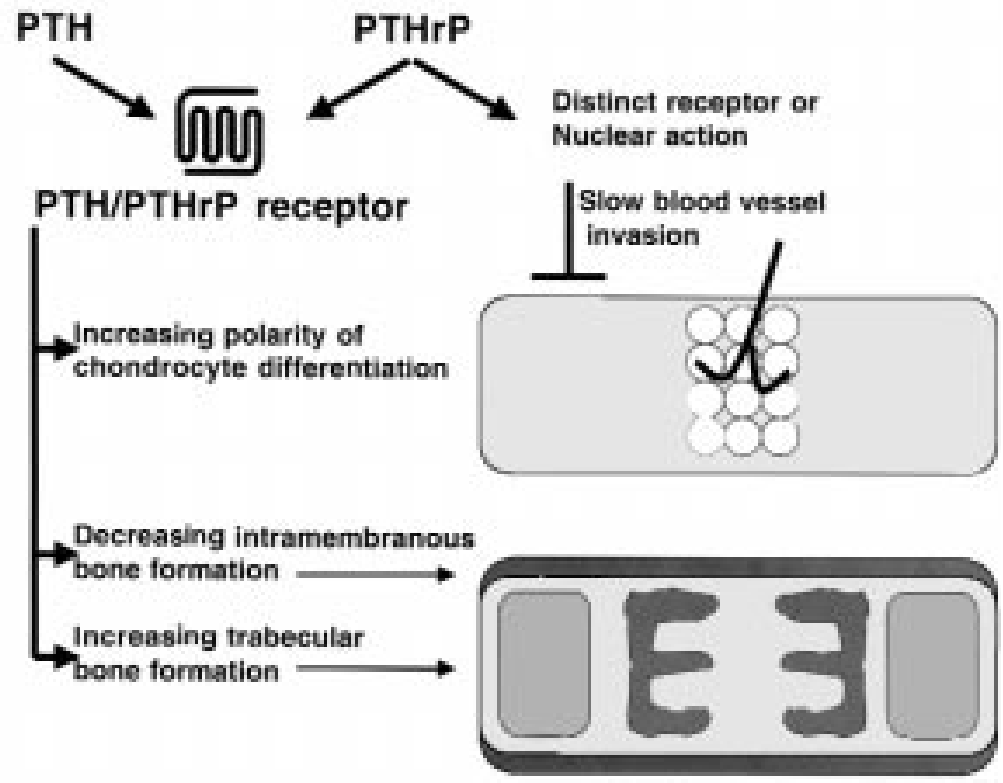


findings in humans with mild primary hyperparathyroidism. This disease is associated with normal or increased bone volume in areas that are primarily trabecular (such as vertebrae), and with loss of bone in cortical areas (32-34). Although this pattern during PTH excess is the expected opposite of that found in the PTH/PTHrP-R $\mathrm{R}^{-/}$ mice, further work will be needed to relate the phenotypes of developing mice to those of adult humans.

In conclusion, we have demonstrated that PTHrP reinforces the polarity of chondrocyte differentiation early in bone development, an action that is mediated through the classical PTH/PTHrP receptor. Furthermore, PTHrP slows blood vessel invasion of the cartilage mold independently of this receptor. In addition, our data support the hypothesis that PTH increases the initial formation of trabecular bone and limits the formation of intramembranous bone adjacent to the cartilage mold of long bones. These contrasts illustrate the complexity of the interactions of PTH, PTHrP, and the PTH/PTHrP receptor.

\section{Acknowledgments}

The authors wish to thank Michael Mannstadt, Ung-il Chung, and Ernestina Schipani for their valuable suggestions, discussions, and technical support. This work was supported by National Institutes of Health grants DK-47038 (to H.M. Kronenberg) and AR-4445354 (to R. Baron). B. Lanske was supported in part by a fellowship from the Max-Kade Foundation.

1. Bringhurst, F.R., Demay, M.B., and Kronenberg, H.M. 1998. Hormones and disorders of mineral metabolism. In Williams textbook of endocrinology. 9th edition. J.D. Wilson, D.W. Foster, H.M. Kronenberg, and P.R. Larsen, editors. W.B. Saunders Co. Philadelphia, PA. 1155-1210.

2. Kream, B., Rowe, D., Gworek, S., and Raisz, L. 1980. Parathyroid hormone alters collagen synthesis and procollagen mRNA levels in fetal rat calvaria. Proc. Natl. Acad. Sci. USA. 77:5654-5658.

3. Yu, X., and Chandrasekhar, S. 1997. Parathyroid hormone (PTH 1-34) regulation of rat osteocalcin gene transcription. Endocrinology. 138:3085-3092

4. Noda, M., Toon, K., and Rodan, G. 1988. Cyclic AMP-mediated stabilization of osteocalcin mRNA in rat osteoblast-like cells treated with parathyroid hormone. J. Biol. Chem. 263:18574-18577.

5. Quinn, C., et al. 1990. Rat collagenase. J. Biol. Chem. 265:22342-22347.

6. Kano, J., et al. 1994. Second messenger signaling of c-fos gene induction by parathyroid hormone (PTH) and $\mathrm{PTH}$-related peptide in osteoblastic osteosarcoma cells: its role in osteoblast proliferation and osteoclast-like cell formation. J. Cell. Physiol. 161:358-366.

7. Bellows, C., Ishida, H., Aubin, J., and Heersche, J. 1990. Parathyroid hormone reversibly suppresses the differentiation of osteoprogenitor cells into functional osteoblasts. Endocrinology. 127:3111-3116.

8. Nishida, S., et al. 1994. Increased bone formation by intermittent parathyroid hormone administration is due to the stimulation of proliferation and differentiation of osteoprogenitor cells in bone marrow. Bone. 15:717-723.

9. Karaplis, A.C., et al. 1994. Lethal skeletal dysplasia from targeted disruption of the parathyroid hormone-related peptide gene. Genes Dev. 8:277-289.

10. Vortkamp, A., et al. 1996. Regulation of rate of cartilage differentiation by Indian hedgehog and PTH-related protein. Science. 273:613-621.

11. Urena, P., et al. 1993. Parathyroid hormone (PTH)/PTH-related peptide $(\mathrm{PTHr})$ receptor $\mathrm{mRNA}$ is widely distributed in rat tissues. Endocrinology. 133:617-623.
12. Orloff, J.J., et al. 1992. Analysis of PTHrP binding and signal transduction mechanisms in benign and malignant squamous cells. Am. J. Physiol. 262:E599-E607.

13. Orloff, J.J., et al. 1995. Further evidence for a novel receptor for aminoterminal parathyroid hormone-related protein on keratinocytes and squamous carcinoma cell lines. Endocrinology. 136:3016-3023.

14. Murray, T.M., Rao, L.G., Muzaffar, S.A., and Ly, H. 1989. Human parathyroid hormone carboxyl terminal peptide (53-84) stimulates alkaline phosphatase activity in dexamethasone-treated rat osteosarcoma cells in vitro. Endocrinology. 124:1097-1099.

15. Murray, T.M., Rao, L.G., and Muzaffar, S.A. 1991. Dexamethasone-treated ROS $17 / 2.8$ rat osteosarcoma cells are responsive to human carboxylterminal parathyroid hormone peptide hPTH(53-84): stimulation of alkaline phosphatase. Calcif. Tissue Int. 49:120-123.

16. Inomata, N., Akiyama, M., Kubota, N., and Jüppner, H. 1995. Characterization of a novel parathyroid hormone (PTH) receptor with specificity for the carboxyl-terminal region of PTH(1-84). Endocrinology. 136:4732-4740.

17. Fukayama, S., Tashiian, A., Jr., Davis, J., and Chisholm, J. 1995. Signaling by $\mathrm{N}$ - and $\mathrm{C}$-terminal sequences of parathyroid hormone-related protein in hippocampal neurons. Proc. Natl. Acad. Sci. USA. 92:10182-10186.

18. Usdin, T.B., Gruber, C., and Bonner, T.I. 1995. Identification and functional expression of a receptor selectively recognizing parathyroid hormone, the PTH2 receptor. J. Biol. Chem. 270:15455-15458.

19. Lanske, B., et al. 1998. The parathyroid hormone/parathyroid hormonerelated peptide receptor mediates actions of both ligands in murine bone. Endocrinology. 139:5194-5204.

20. Lanske, B., et al. 1996. PTH/PTHrP receptor in early development and Indian-hedgehog-regulated bone growth. Science. 273:663-666.

21. Kovacs, C.S., et al. 1996. Parathyroid hormone-related peptide (PTHrP) regulates fetal-placental calcium transport through a receptor distinct from the PTH/PTHrP receptor. Proc. Natl. Acad. Sci. USA. 93:15233-15238.

22. Lee, K., Deeds, J.D., and Segre, G.V. 1995. Expression of parathyroid hormone-related peptide and its receptor messenger ribonucleic acid during fetal development of rats. Endocrinology. 136:453-463.

23. Lee, K., et al. 1994. Parathyroid hormone induces sequential $c$-fos expression in bone cells in vivo: in situ localization of its receptor and $c$-fos messenger ribonucleic acids. Endocrinology. 134:441-450.

24. Lee, K., et al. 1996. Parathyroid hormone-related peptide delays terminal differentiation of chondrocytes during endochondral bone development. Endocrinology. 137:5109-5118.

25. Amling, M., et al. 1997. Bcl-2 lies downstream of parathyroid hormonerelated peptide in a signaling pathway that regulates chondrocyte maturation during skeletal development. J. Cell Biol. 136:205-213.

26. Hahn, M., Vogel, M., and Delling, G. 1991. Undecalcified preparation of bone tissue: report of technical experience and development of new methods. Virchows Arch. A Pathol. Anat. Histopathol. 418:1-4.

27. Lee, K., et al. 1996. Localization of parathyroid hormone/parathyroid hormone-related peptide receptor mRNA in kidney. Am. J. Physiol. 270:F186-F191.

28. Amizuka, N., Warshawsky, H., Henderson, J.E., Goltzman, D., and Karaplis, A.C. 1994. Parathyroid hormone-related peptide-depleted mice show abnormal epiphyseal cartilage development and altered endochondral bone formation. J. Cell Biol. 126:1611-1623.

29. Orloff, J.J., et al. 1996. A midregion parathyroid hormone-related peptide mobilizes cytosolic calcium and stimulates formation of inositol triphosphate in a squamous carcinoma cell line. Endocrinology. 137:5376-5385.

30. Orloff, J.J., et al. 1995. A novel receptor for amino-terminal parathyroid hormone-related protein on keratinocytes and squamous carcinoma cells lines. Endocrinology. 136:3016-3025.

31. Henderson, J.E., et al. 1995. Nucleolar localization of parathyroid hormone-related peptide enhances survival of chondrocytes under conditions that promote apoptotic cell death. Mol. Cell. Biol. 15:4064-4075.

32. Heersche, J., Bellows, C., and Aubin, J. 1994. Cellular actions of parathyroid hormone on osteoblast and osteoclast differentiation. In The parathyroids. J. Bilezikian, M. Levine, and R. Marcus, editors. Raven Press. New York, NY. 83-91.

33. Grey, A. 1997. The skeletal effects of primary hyperparathyroidism. Baillieres Clin. Endocrinol. Metab. 11:101-116.

34. Silverberg, S.J., et al. 1989. Skeletal disease in primary hyperparathyroidism. J. Bone Miner. Res. 4:283-291. 